\title{
Effectiveness of Subcutaneous Negative-pressure Drain in Cases of Infective Laparotomies
}

\author{
Ankit Goyal $^{1}$, Rajkamal Kanojiya ${ }^{2}$
}

\begin{abstract}
Wound infection is the most common complication in patients who underwent infective laparotomies, leading to postoperative complications, pus discharge, prolonged hospitalization, increased cost of treatment, etc. Even after sensitive-based antibiotic use and judicious irrigation of wound with saline, it remained a major concern.

Aims and objectives: To evaluate the role of closed suction drainage in reduction of postoperative wound infection in cases of infective laparotomies when compared to laparotomy wounds without closed suction drainage. To evaluate whether negative-pressure closed suction drainage is effective in reducing the hospital stays. To evaluate whether negative-pressure closed suction drainage is effective in reducing the cost of treatment.

Materials and methods: This study is conducted on patients who underwent infective laparotomies and we divided it into two groups: one with subcutaneous negative-pressure drain in and other without it.

Conclusion: Closed suction drain prevented postoperative wound infection significantly, reduces hospital stay, reduces cost of treatment, and helped in early healing of wound and removal of sutures.

Keywords: Discharge, Drain, Healing, Sepsis, Wound infection.

Journal of Mahatma Gandhi University of Medical Sciences \& Technology (2019): 10.5005/jp-journals-10057-0099
\end{abstract}

\section{INTRODUCTION}

In humans, regeneration of tissues is limited. Only epithelium and liver can actually regrow. Most tissues heal by repair, which results in scarring. ${ }^{1,2}$

Infections that occur in wound created by any invasive surgical procedure are generally known as surgical site infections (SSIs). ${ }^{3,4}$ SSI previously termed as postoperative wound infection is defined as the infection presented 30 days after the surgical procedure if no prosthesis is being implanted; and up to 1 year, if a prosthesis is implanted in the patient's body. ${ }^{5,6}$ The incidence of SSI increases with the increase in abdominal contamination.

Surgical or operative site infection is also one of the most common operative complication, occurring in about $5 \%$ of all patients who underwent surgery, and this ratio is, more upto $30-40 \%$, in those patients undergoing abdominal surgery, depending on the level of contamination. ${ }^{\text {? }}$

Development of an SSI has a large impact on mortality, morbidity healthcare costs, patient inconvenience, and dissatisfaction. ${ }^{8,9}$ Infectious complications are the main causes of postoperative morbidity in abdominal surgery wound infection. The most common form is superficial wound infection occurring within the first week of surgery. ${ }^{10}$ Abdominal wall closure with existence of infection in it is a challenge.

Surgical infections are characterized by a breach of mechanical or anatomic defense mechanisms and are associated with greater morbidity, significant mortality, and increased health care cost. ${ }^{11}$ Usually, such patients present late after hours, or even after days of perforation, bowel wall is thickened with edema with sepsis in peritoneum causing leakage of fluid, sometimes even pus, till control of infection. After evaluation and management pathology and peritoneal cleaning, closure done under tension may cause compartment syndrome or burst abdomen causing wound

\footnotetext{
1,2Department of General Surgery, Mahatma Gandhi Medical College and Hospital, Sitapura, Jaipur, Rajasthan, India
}

Corresponding Author: Ankit Goyal, Department of General Surgery, Mahatma Gandhi Medical College and Hospital, Sitapura, Jaipur, Rajasthan, India, Phone: +91 9001714000, e-mail: dr.goyalankit@ gmail.com

How to cite this article: Goyal A, Kanojiya R. Effectiveness of Subcutaneous Negative-pressure Drain in Cases of Infective Laparotomies. J Mahatma Gandhi Univ Med Sci Tech 2019;4(2): 35-39.

Source of support: Nil

Conflict of interest: None

dehiscence in a significant number of patients. ${ }^{12}$ It has been postulated that if there is presence of hematoma, serous fluid, and dead space in surgical wound, it can increase the risk of infection as this acts as a good culture medium. ${ }^{13,14}$ Prevalence of SSI may be partially explained by the emergence of antimicrobial resistant pathogens and the increasing number of surgical interventions among elderly and/or a wide variety of chronic, debilitating, or immune compromising underlying diseased patients. ${ }^{15} \mathrm{SSI}$ can double the length of hospital stay and that increases hospital cum healthcare costing. Seroma can lead to wound-related complication, such as abscess, calcified seroma, poor cosmesis, and mal appearance of scar. ${ }^{16}$ Different material sutures have been used by surgeons for wound closures, either absorbable or nonabsorbable sutures. ${ }^{17}$ Negative suction in subcutaneous space has shown to decrease possibility of infection by removal of infectious content and evacuation of collected seroma in subcutaneous space. Negative suctioning causes improvement in healing of wound by decreasing its microbial load. ${ }^{18-20}$ 


\section{Aim and Objectives}

- To evaluate the role of closed suction drainage in reduction of postoperative wound infection in cases of infective laparotomies when compared to laparotomy wounds without closed suction drainage.

- To evaluate whether negative-pressure suction drain is effective in reducing the hospital stay.

- To evaluate whether negative-pressure suction drain is effective in reducing the cost of treatment.

- To evaluate whether closed suction drainage is beneficial in increasing patient's comfort and decreasing patient's morbidity.

- To study the epidemiology of wound infection.

- To evaluate the type of healing, primary or secondary.

\section{Materials and Methods}

Type of Study

Prospective randomized controlled trials

\section{Study Period}

January 2018 to June 2019

\section{Place of Study}

Department of General Surgery, Mahatma Gandhi Medical College and Hospital, Jaipur.

This study was conducted on 60 patients. Patients were chosen randomly irrespective of their age and sex. One control group included 30 cases, and the other study group comprised of other 30 cases (Fig. 1).

All details about the surgery/operation were being noted, and operative wounds were classified according to definition being given by the American College of Surgeons as:

- Clean

- Clean contaminated

- Contaminated

- Dirty

At the time to abdominal wall closure, first of all the peritoneum was sutured/closed appropriately with absorbable suture material, and then rectus sheath was closed by nonabsorbable suture material continuously like PDS loop. ${ }^{21}$

One or more intraperitoneal drains were placed inside-in potential areas such as pelvis/subhepatic/peri-splenic. A tube with multiple holes small in size was positioned with its tip lying extraperitoneal over the rectus sheath, and it was brought out through area of healthy skin by a different incision away from the wound site and fixed with nonabsorbable suture material and connected with a closed suction drain. Incision was then closed with non-absorbable suture, and aseptic sterile dressing was done.

First dressing was changed after 48 hours, and incision site was closely monitored for pain, tenderness, induration, redness, discharge, swelling, increased local warmth, and suture with tension (Table 1).

Discharge was collected and sent for culture and sensitivity.

Criteria of wound infection:

Wound infection is described as:

- Incisional-When it is located above the fascial layer

- Deep-When it is located below the fascial layer.

Types of discharges through the wound are:

- Serous

- Seropurulent

- Purulent

- Fecopurulent.

Drain was taken out when the output was $5 \mathrm{~mL}$ or less. The sutures were cut and taken out either before or sometimes after the discharge of the patient at least after 8 days depending upon the wound site condition. Patient was followed up in the hospital outpatient department or through correspondence by phone/email/messages/social media for up to 30 days postoperatively.

Statistical analysis was made to find significant association.

\section{Observation and Results}

The study was conducted on 60 patients -30 with negativepressure subcutaneous drain and 30 without drain.

Relevant findings noted in this study are highlighted here.

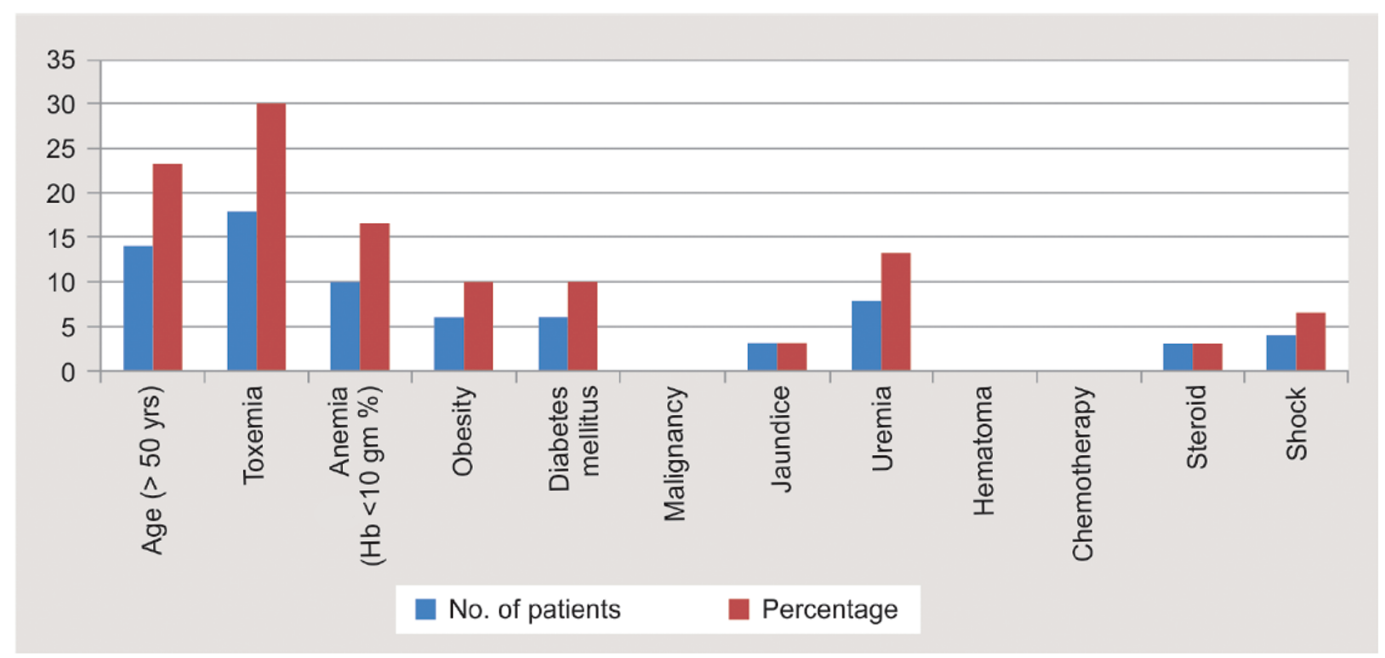

Fig. 1: Associated risk factors 


\section{Operative Procedure}

Most common operative procedure was primary repair (65\%) followed by resection anastomosis (15\%), ileostomy (13.33\%), appendicectomy in $5 \%$, and colostomy $(1.66 \%)$, respectively (Table 1).

The comparison in our study between groups, that is control without closed suction drain and study group with closed suction drain, gave result that discharge from wound in the study group compared to discharge from the wound in control study group is significantly high with $p$ value of 0.0195 (Table 2).

\section{Associated Risk Factors}

The comparison in our study between the groups, that iscontrol without closed suction drain and study group with closed suction drain, gave result that postoperative day of detection of wound infection among the group that used closed suction drain when compared to the group without closed suction drain is statistically highly significant with $p$ value of 0.0099 . It clarifies that closed suction drain is very effective in preventing the wound infection, especially in much prone first four postoperative days (Table 2).

The comparison in our study between the groups, that is control without closed suction drain and study group with closed suction drain, gave result that discharge from stitch line among the group that used closed suction drain when compared to the

Table 1: Surgical wound infection sign and symptoms

\begin{tabular}{lcll}
\hline Features & Control group & Study group & Total \\
\hline Pain & 13 & 7 & 20 \\
Tenderness & 12 & 7 & 19 \\
Redness & 8 & 5 & 13 \\
Discharge & $\mathbf{1 8}$ & $\mathbf{9}$ & $\mathbf{2 7}$ \\
Raised local & 8 & 4 & 12 \\
$\begin{array}{l}\text { temperature } \\
\begin{array}{l}\text { Suture under tension/ } \\
\text { cut through }\end{array}\end{array}$ & 5 & 3 & 8 \\
\hline
\end{tabular}

Table 2: Postoperative day of detection of wound infection control vs study

\begin{tabular}{llcll}
\hline Duration & Control & Study & Total & $p$ value \\
\hline$\leq 4$ days & 13 & 4 & 17 & 0.0099 \\
$>4$ days & 17 & 26 & 43 & \\
Total & 30 & 30 & 60 & \\
\hline
\end{tabular}

Table 3: Discharge from stitch: control vs study

\begin{tabular}{llcll}
\hline Duration & Control & Study & Total & p value \\
\hline$\leq 4$ days & 13 & 4 & 17 & 0.0099 \\
$>4$ days & 17 & 26 & 43 & \\
Total & 30 & 30 & 60 & \\
\hline
\end{tabular}

Table 4: Removal of drain: control vs study

\begin{tabular}{lclll}
\hline $\begin{array}{l}\text { Removal of } \\
\text { drain }\end{array}$ & Control & Study & Total & p value \\
\hline$\leq 4$ days & 4 & 12 & 16 & 0.0023 \\
$>4$ days & 26 & 18 & 44 & \\
Total & 30 & 30 & 60 & \\
\hline
\end{tabular}

group without closed suction drain gave result that only 9 of 30 patients in the study group developed stitch line infection, whereas 18 of 30 among control group developed stitch line infection and statistically is significantly high having a $p$ value of 0.0195 . It proves that closed suction drain is very effective in preventing the discharge from wound infection postoperative days (Table 3 ).

In this study, we found that in patients of this study group, abdominal collection and its abdominal drain was removed early as assessed with another group in the study. The results are significantly high with $p$ value of 0.0023 (Table 4).

In this study, we found that in the patient group in which negative-pressure suction drain was used a very early wound healing was seen when compared to the control in which drain was not used.

In this study group, 27 of 30 patients had their wound healed, so sutures were removed early within 10 days compared to control group where only 13 of 30 patients showed wound healing signs and only sutures were taken out. This comparison gave a very highly significant statistical data with $p$ value of 0.0001 (Table 5).

In this study, we found that patients with negative-pressure drain have a early recovery compared to the control group where negative-pressure drain was not used. Due to this, 17 of 30 patients recovered well without signs of infection and were discharged early within 10 days compared to control group in who only 4 of 30 patients showed recovery signs and were discharged within first 10 postoperative days. This comparison gave a very highly significant statistical data with $p$ value of 0.0004 (Table 6).

In this study, we found that patients with closed suction drain have significantly more number of by primary intention/closure compared to patients in the control group in who closed suction drain was not used. As a result, 27 of 30 patients in the study group healed by primary intention/closure compared to control group where only 19 of 30 patients showed healing by primary intention. This comparison gave a very highly significant statistical data with a $p$ value of 0.0146 (Table 7).

Table 5: Removal of sutures: control vs study

\begin{tabular}{lllll}
\hline $\begin{array}{l}\text { Removal of } \\
\text { Sutures }\end{array}$ & Control & Study & Total & p value \\
\hline$\leq 10$ days & 13 & 27 & 40 & 0.0001 \\
$>10$ days & 17 & 3 & 20 & \\
Total & 30 & 30 & 60 & \\
\hline
\end{tabular}

Table 6: Average duration of hospital stay (in days) control vs study

\begin{tabular}{lclll}
\hline $\begin{array}{l}\text { Removal of } \\
\text { sutures }\end{array}$ & Control & Study & Total & p value \\
\hline$\leq 10$ days & 4 & 17 & 21 & 0.0004 \\
$>10$ days & 26 & 13 & 39 & \\
Total & 30 & 30 & 60 & \\
\hline
\end{tabular}

Table 7: Healing by primary/secondary intention: control vs study

\begin{tabular}{lllll}
\hline Healing & Control & Study & Total & pvalue \\
\hline $\begin{array}{l}\text { Primary } \\
\text { intention }\end{array}$ & 19 & 27 & 46 & 0.0146 \\
$\begin{array}{l}\text { Secondary } \\
\text { intention }\end{array}$ & 11 & 3 & 14 & \\
Total & 30 & 30 & 60 & \\
\hline
\end{tabular}


We were able to reduce rate of wound infection rate by using closed suction drain in infective laparotomies. By reducing the rate of wound infection rate, we were also able to help wound healing by primary intention in about $90 \%$ of patients in the study group compared to $63.33 \%$ in the control group of infective laparotomies.

\section{Discussion}

Surgical wound infection continued to be the major cause for morbidity as well as mortality in clinics, despite perioperative adequate coverage of antibiotics. Although thorough control of wound infection is not feasible, reduction in infection rate to a lower level could have much benefits for both patients easiness and human/material resources in use.

Healing has been parted into phases for purposeful description: phase of inflammation, proliferation, and maturation.

Negative suction pressure helps in wound healing and its benefits are as follows:

\section{Protection}

By covering the wound primarily, a packed wound environment is produced. This is beneficial, as it protects the wound from environmental contaminations.

\section{Blood Flow}

Both clinically and experimentally, the application of subatmospheric pressure removes third space fluid from the area immediately adjacent to the wound. Removal of this fluid results in a decrease in tissue turgor and a decrease in capillary after load, which promotes better capillary circulation and better inflow. Adequacy of the circulation supports wound moisture. Additionally, the removal of excess fluid is thought to remove inhibitory factors present in the fluid.

\section{Decrease in Bacterial Load}

Concomitant with the rise in blood flow and removal of stagnancy of fluid in wound, which is very good media for microbes, bacterial load is reduced. This is evidenced by a decrease in superficial purulence, slime production, and odor. Raise of oxygen and perfusion in compromised and damaged tissues enhances the resistance to infection (Bunt TK, 1998).

\section{Increased Granulation Tissue Proliferation}

It is significantly higher in wounds managed with negative pressure application and is significantly higher than the rates reported for human growth factors and saline dressing-treated wounds (Morykwas MJ 1997). This significantly high rate of increment is due to the transmission of uniformly applied force on the wound periphery. Application of negative pressure changes the external forces, bridges, and cytoskeletons, thus releasing intracellular second messengers (PGs, Inositol phosphates, PKinase C and Ca ${ }^{++}$) (Ingber D, 1991). Negative pressure gets the wound heal faster. Studies proved mechanical stretching allows the cells to proliferate at a faster pace. Irritation, continuously by negative suction pressure, dramatically increases the rate of regrowth of the tissues.

\section{Wound Contraction}

Negative pressure when applied to the wound contracts. The suction drain applies a pressure of approximately 100 to 200 $\mathrm{mm} \mathrm{Hg}$ below atmospheric pressure. Edema fluid is removed from the interstitial spaces that eliminate an extrinsic reason for microcirculatory deterioration and improves blood supply, local oxygenation, and promoting angiogenesis. The mechanical tension from the vacuum futher directly stimulates cell proliferation and granulation. It also exerts a force of mechanical nature on the tissues and attracts the wound edges toward it.

\section{ConCLUSION}

This study concludes that placement of a subcutaneous negativepressure drain in infective laparotomies reduces wound infection, helps in early postoperative recovery, better wound healing, and decreases financial burden on patient by decreasing hospital stay and infection.

Therefore, this method of closed suction drainage is very strongly recommended for the prevention of wound infection in infective laparotomies.

\section{References}

1. McDonald K, Romano P, Davies S, et al. Postoperative wound dehiscence: number of abdomino-pelvic surgery patients with disruption of abdominal wall per 1,000 eligible admissions.

2. Farber BF, Wenzel RP. Postoperative wound infection rates: Results of prospective statewide surveillance. Am J Surg 1980;140(3):343-346.

3. Shah B, Singh AK, Sheikh SS. Role of closed suction drain in prevention of suture line infection in elective laparotomy wounds. Imp J Interdiscip Res (IJIR) 2016;2(6):1ISSN 2454-1362.

4. Smyth ET, Mcllvenny G, Enstone JE. Four country healthcare associated Infection prevalence survey 2006. J Hospital Infect 2008;69(3):230-248. DOI: 10.1016/j.jhin.2008.04.020.

5. Gupta P, Kumar R. Role of subcutaneous suction drain in reducing site infections after emergency laparotomy. Inter Surg J 2017;4(8). DOI: 10.18203/2349-2902.isj20173141.

6. Awad SS, Palacio $\mathrm{CH}$, Subramanian A, et al. Implementation of a methicillin-resistant Staphylococcus aureus (MRSA) prevention bundle results in dcreased MRSA surgical site infections. Am J Surg 2009;198(5):607-610. DOI: 10.1016/j.amjsurg.2009.07.010.

7. Bruce J, Russell EM, Millinson J, et al. The measurement and monitoring of surgical adverse events. Health Tech Assess 2001;5(22):1-194. DOI: $10.3310 /$ hta5220.

8. Wilson APR, Gibbons C, Reeves BC, et al. Surgical wound infection as a performance indicator: agreement of common definitions of wound infection in 4773 patients. BMJ 2004;329(7468):720-724. DOI: 10.1136/bmj.38232.646227.DE.

9. Scott RDII. The direct medical costs of healthcare-associated infections in U.S. hospitals and the benefits of prevention. Division of Healthcare Quality Promotion, National Center for Preparedness, Detection, and Control of Infectious Diseases, Coordinating Center for Infectious Diseases, Centers for Disease Control and Prevention, March (2009). Available at: http://www.cdc.gov/hai/pdfs/hai/ scott_costpaper.pdf.

10. Kujath $P$, Kujath C. Complicated skin, skin structure and soft tissue infections - are we threatened by multi-resistant pathogens? Eur J Med Res 2010;15(12):544-553. DOI: 10.1186/2047-783x-15-12-544.

11. Leaper DJ. Surgical infection. Bailey \& Love's Short Pract Surg 2004.

12. Vashist M, Singla A, Malik V, et al. Abdominal wall closure in the presence of sepsis: role of negative suction. Int J Surg 2013;29:1.

13. Manzoor B, Heywood N, Sharma A. Surgery Research and Practice. Hindawi Publishing Corporation, vol. 201, 2015. p. 715803. DOI: 10.1155/2015/715803.

14. Drains. Dead Space Management in Complications in Surgery Mulholland MW, Doherty GM, ed. 2nd ed., Philadelphia, Pa., USA: Wolters Kluwer, Lipincott William \& Wilkins Health; 2011. p. 148.

15. Richard T. Wound healing. Sabiston Text book of Surgery. 19th ed., 2012. pp. 151-177.

16. Corman ML, Veidenheimer MC, Coller JA. Controlled clinical trial of three suture materials for abdominal wall closure after bowel 
operations. Am J Surg 1981;141(4):510-513. DOI: 10.1016/00029610(81)90150-1.

17. Morykuwas MJ, Argenta LC. The use of negative pressure drain to increase the rate of granulation tissue formation in chronic open wounds. Annual meeting federation of American societies for experimental biology, New Orleans 1993.

18. Sadoshima J, Izumo S. Mechanical stretch rapidly activates multiple signal transduction pathways in cardiac myocytes:potential involvement of an autocrine/paracrine mechanism. EMBO J 1993;12:1681-1692.

19. Vandenburgh $\mathrm{HH}$. Mechanical forces and their second messengers in stimulating cell growth in vitro. Am J Physiol 1992;262:350-355.

20. Wirtz HR,Dobbs LG. Calcium mobilization and exocytosis after one mechanical stretch of lung epithelial cell. Science 1990;250:266-269.

21. Maingoat's abdominal operation, 13th edition, Michael J Zinner/ Stanley W. Ashley; II-10/480. 